\title{
Involuntary Orienting and Conflict Resolution during Auditory Attention: The Role of Ventral and Dorsal Streams
}

\author{
Hannah J. Stewart ${ }^{1,2,3}$, Dawei Shen ${ }^{1}$, Nasim Sham ${ }^{1}$, and Claude Alain ${ }^{1,4}$
}

\begin{abstract}
Selective attention to sound object features such as pitch and location is associated with enhanced brain activity in ventral and dorsal streams, respectively. We examined the role of these pathways in involuntary orienting and conflict resolution using fMRI. Participants were presented with two tones that may, or may not, share the same nonspatial (frequency) or spatial (location) auditory features. In separate blocks of trials, participants were asked to attend to sound frequency or sound location and ignore the change in the task-irrelevant feature. In both attend-frequency and attend-location tasks, RTs were slower when the task-irrelevant feature changed than when it stayed the same (involuntary orienting). This behavioral cost coincided with enhanced activity in the $\mathrm{pFC}$ and superior temporal gyrus. Conflict resolution was examined by comparing
\end{abstract}

\section{INTRODUCTION}

To navigate successfully in complex auditory environments, the listener needs to identify "what" different sounds are and "where" they are coming from (Alain, Arnott, Hevenor, Graham, \& Grady, 2001; Alain \& Arnott, 2000). Evidence from animal studies (Lomber \& Malhotra, 2008; Rauschecker \& Tian, 2000; Romanski et al., 1999; Rauschecker, 1998) as well as from fMRI studies (Leung \& Alain, 2011; Alain, He, \& Grady, 2008; Degerman, Rinne, Salmi, Salonen, \& Alho, 2006; Alain et al., 2001; Maeder et al., 2001) suggest that ventral and dorsal brain areas play an important role in identifying and locating sounds in the environment, respectively. Human lesion studies have shown double dissociations between nonspatial and spatial auditory processing in patients with left- and right-hemisphere damage (Zündorf, Lewald, \& Karnath, 2016; Clarke \& Thiran, 2004; Clarke et al., 2002; Clarke, Bellmann, Meuli, Assal, \& Steck, 2000). Meta-analyses of auditory fMRI studies provide further support for ventral and dorsal pathways involved in processing the sound object identity and location,

\footnotetext{
${ }^{1}$ Baycrest Centre, Toronto, Ontario, Canada, ${ }^{2}$ University College London, ${ }^{3}$ Cincinnati Children's Hospital Medical Center, ${ }^{4}$ University of Toronto
}

situations where the change in stimulus features was congruent (both features changed) and incongruent (only one feature changed). Participants were slower and less accurate for incongruent than congruent sound features. This congruency effect was associated with enhanced activity in the $\mathrm{pFC}$ and was greater in the right superior temporal gyrus and medial frontal cortex during the attend-location task than during the attend-frequency task. Together, these findings do not support a strict division of "labor" into ventral and dorsal streams but rather suggest interactions between these pathways in situations involving changes in task-irrelevant sound feature and conflict resolution. These findings also validate the Test of Attention in Listening task by revealing distinct neural correlates for involuntary orienting and conflict resolution.

respectively (Alho, Rinne, Herron, \& Woods, 2014; Arnott, Binns, Grady, \& Alain, 2004).

Whereas a number of imaging studies have investigated the dual-pathway theory with varying types of auditory stimuli and tasks (e.g., noise bands with center frequencies, Alain et al., 2001; dichotic vowel identification, Du et al., 2015; and three-talker sentences, Hill \& Miller, 2010), there have been few investigations into the auditory ventral and dorsal pathways with regard to specific attentional constructs (e.g., Orr \& Weissman, 2009; Mayer, Harrington, Adair, \& Lee, 2006). The few that do explore different attentional constructs assess each construct in isolation, despite multiple attentional constructs being used in parallel in real life. Zhang, Barry, Moore, and Amitay (2012) have developed a test, the Test of Attention in Listening (TAiL), based on two of Posner and Petersen's (1990; Petersen \& Posner, 2012) attention network constructs. During auditory attention tasks, TAiL can isolate involuntary orienting (attending to a taskirrelevant property/object) and executive control (completing the dominant task while also performing a subdominant task, e.g., conflict resolution).

Orienting to auditory information can occur voluntarily via cues or be triggered involuntarily by unexpected novel sound events. Cues, whether valid or invalid, exert strong effects on behavior and cortical responses (Mayer, 
Franco, \& Harrington, 2009; Posner \& Petersen, 1990). The TAiL paradigm does not involve cues and so focuses on involuntary orienting. This attention construct has been widely investigated through auditory oddball paradigms where an infrequent uncued stimuli change occurs (e.g., loudness, pitch, location) to elicit attentional capture. However, in the TAiL paradigm, the frequency (pitch) and location changes are subtle and occur in every trial. Neuroimaging of such a paradigm is novel.

Two articles using auditory stimuli similar to ours, but in an oddball task, compared cortical areas activated when involuntarily orienting to task-irrelevant stimuli (loudness deviant) during nonspatial (pitch: Alho, Salmi, Koistinen, Salonen, \& Rinne, 2015) and spatial (location: Salmi, Rinne, Koistinen, Salonen, \& Alho, 2009) task-relevant paradigms. They found that the two paradigms showed overlapping areas of activation in the posterior STS, middle temporal gyrus (MTG), and middle frontal gyrus (MFG). However, there was stronger bilateral activation in large areas of the superior temporal gyrus (STG) and STS in the nonspatial task compared to the spatial task and in the ventromedial prefrontal area for the opposite contrast. Loudness was used to trigger involuntary orienting in both of the paradigms. As evaluating the use of the dual pathways was not a goal of these sister articles, it is still unclear whether involuntarily orienting to nonspatial and spatial auditory information would be associated with segregation or overlapping of the ventral and dorsal streams.

A classic methodology for testing conflict resolution involves contrasting incongruent with congruent trials, as in the Stroop task (Stroop, 1935). Roberts and Hall (2008) conducted a meta-analysis of the visual Stroop task and found evidence of a common frontoparietal network, including ACC, inferior frontal gyrus (IFG), parietal lobe, and anterior insula. They followed this meta-analysis up with the same group of participants completing the traditional visual Stroop task and an auditory version where listeners indicated whether the speaker's voice was high or low in pitch, while ignoring semantic content (the words "high," "low," and "day"). The findings from the fMRI study were consistent with the results of their meta-analysis with a common frontoparietal network for both paradigms. However, they also observed extensive activity in the left lateral pFC during the auditory Stroop task along with activity in the STS, and the visual Stroop task showed greater activity in the right IFG. These results suggest a supramodal conflict resolution network with further areas activated for the separate modalities. However, the pathways activated by auditory spatial and nonspatial conflict resolution tasks have not been investigated. Siemann, Herrmann, and Galashan (2018) investigated this question with visual stimuli and found comparable networks were activated for visual spatial and nonspatial conflict resolution (dorsal frontoparietal networks in the left MFG, superior parietal lobule, and FEFs), with additional ventral areas recruited specifically for nonspatial conflict resolution (left MTG, cuneus, and lingual gyrus).
Behavioral studies using TAiL provide support that the task taps into these two attentional constructs of involuntary orientation and conflict resolution using auditory stimuli (Stewart \& Amitay, 2015; Zhang et al., 2012). Using scalp recordings of ERPs, Stewart, Amitay, and Alain (2017) showed distinct ERP modulations associated with involuntary orienting and conflict resolution. The comparison of distributed source analyses for involuntary orienting suggests a more dorsal source when the taskirrelevant sound feature was location than when it was frequency. Although this finding appears consistent with the dual-pathway model of auditory attention, further research is needed to more precisely identify the brain areas associated with involuntary orienting and conflict resolution during the TAiL task.

This current study combines fMRI, with its advantageous spatial resolution, and the TAiL task to investigate the brain areas associated with involuntary orienting and conflict resolution. The paradigm used provides a novel approach to this question as it allows both attentional constructs to be investigated using the same auditory trials and without the sustained attention effects of an oddball paradigm. It is expected that optimum task performance may differentially involve ventral and dorsal streams to process nonspatial and spatial auditory stimulus features, respectively. As found by Alho et al. (2015), we anticipate that involuntary orienting will engage a similar attention network for both spatial and nonspatial tasks including the STS/STG, MTG, and MFG. Because of the paradigm differences between TAil's continuous subtle task-irrelevant changes and previous studies' deviant salient task-irrelevant changes, it is unclear which cortical areas will show additional activity for spatial and nonspatial stimuli. After meta-analyses of the dual-pathway theory of auditory tasks (Alho et al., 2014; Arnott et al., 2004), additional inferior parietal lobe activity is predicted for spatial stimuli; and middle STG activity, for nonspatial stimuli. Conflict resolution contrasts are predicted to show frontoparietal activation including ACC, IFG, and parietal lobe, as found by Roberts and Hall (2008) and Siemann et al. (2018). However, it is unclear whether larger supramodal or auditory-specific networks will be engaged for the spatial (e.g., frontal cortex) and nonspatial (e.g., amodal: MTG; supramodal: cuneus and lingual gyrus) auditory stimuli.

\section{METHODS \\ Participants}

Seventeen right-handed participants aged $19-30$ years ( $M=24.74$ years, $S D=3.43$ years; 13 women and four men) were recruited through the Rotman Research Institute participant database. Inclusion criteria were normal hearing (thresholds below $25 \mathrm{~dB}$ hearing level bilaterally at frequencies between 250 and $8000 \mathrm{~Hz}$, inclusive) and a normal score (0-3) on the Quick Speech-in-Noise test (Killion, Niquette, Gudmundsen, Revit, \& Banerjee, 2004). 


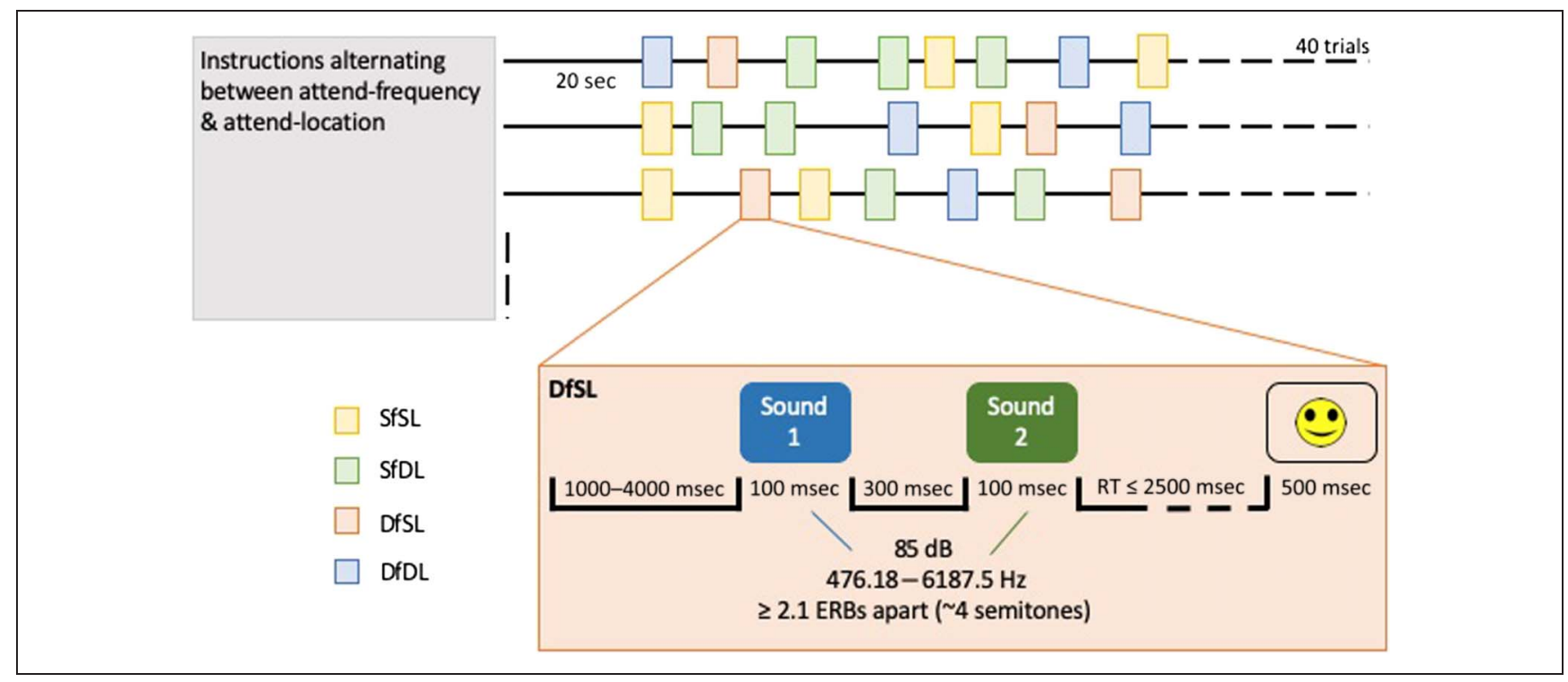

Figure 1. Paradigm schematics of runs and a single (DfSL) trial. Twelve runs were made up of alternating attend-frequency and attend-location TAiL tasks, counterbalanced across participants. Within each run, 40 trials were presented consisting of 10 each of SfSL, SfDL, DfSL, and DfDL trials randomized for each participant.

Exclusion criteria were any self-reported history of brain damage, brain surgery, language- or attention-related conditions, autism spectrum disorders, or any auditory system disorders. All methods were approved by the research ethics board at Baycrest Health Sciences and performed in accordance with the relevant guidelines and regulations of Toronto Academic Health Services Network. All participants signed informed consent before the experiment and received monetary compensation for their participation.

\section{Stimuli and Task}

All tones were made up of sinusoids with a duration of $100 \mathrm{msec}$, gated on/off by 10-msec cos ramps, and were presented monaurally at about $85 \mathrm{~dB}$ sound pressure level (root mean square) by means of circumaural, fMRIcompatible headphones (Avotec), acoustically padded to suppress scanner noise by about $25 \mathrm{~dB}$ sound pressure level. Tone frequency was randomly selected for each trial from the range of $476.18-6187.50 \mathrm{~Hz}$ with at least 2.1 equivalent rectangular bandwidths ( $\sim 4$ semitones) between the trial's tones, therefore making it well within the listener's ability to discriminate between different frequencies (Jensen \& Neff, 1993). The intertrial interval varied randomly between 1000 and 4000 msec (1000-msec steps, rectangular distribution), whereas the ISI was set at 300 msec (Figure 1).

In both attend-frequency and attend-location tasks, the stimuli and paradigm remained the same, and only the instructions to the participants changed. In each trial, participants heard a tone pair where the individual tones were either the same or different in frequency and/or spatial location (ear presentation; SfSL $=$ same frequency and same location; DfSL = different frequency but same location; $\mathrm{SfDL}=$ same frequency but different location; DfDL $=$ different frequency and different location). In both attend-frequency and attend-location tasks, the listener had to indicate via a button press if the taskrelevant sound feature (i.e., the location of the two tones in the attend-location task) were the same or different, while ignoring the task-irrelevant sound feature (i.e., the frequency of the two tones in the attend-location task). A full description of the task and stimuli can be found in Zhang et al. (2012).

Listeners were asked to respond as fast and accurately as possible after the second tone. Responses less than $200 \mathrm{msec}$ and more than $2500 \mathrm{msec}$ were excluded from further analysis in case of premature responding and interruption of performance. Participants' responses were registered using an fMRI-compatible response pad

Table 1. Calculations for the TAiL Outcome Measures

\begin{tabular}{llc}
\hline & Attend-frequency & Attend-location \\
\hline Baseline & SfSL & SfSL \\
Involuntary orienting & (DfDL + SfDL - DfSL - SfSL $)$ & $($ DfDL + DfSL - SfDL - SfSL) \\
Conflict resolution & (DfSL + SfDL - DfDL - SfSL $)$ & \\
\hline
\end{tabular}


Table 2. RT and Accuracy for the Four Trial Types in Each TAiL Task

\begin{tabular}{|c|c|c|c|c|c|}
\hline \multirow[b]{2}{*}{ Conditions } & & \multicolumn{2}{|c|}{$R T$ (msec) } & \multicolumn{2}{|c|}{ Accuracy (\%) } \\
\hline & & Mean & SEM & Mean & SEM \\
\hline \multirow[t]{4}{*}{ Attend-frequency } & SfSL & 492.41 & 23.51 & 98.60 & 0.79 \\
\hline & SfDL & 675.30 & 38.30 & 91.95 & 2.67 \\
\hline & DfSL & 627.38 & 37.62 & 94.80 & 1.60 \\
\hline & DfDL & 646.55 & 37.93 & 94.54 & 1.36 \\
\hline \multirow[t]{4}{*}{ Attend-location } & SfSL & 517.06 & 25.44 & 97.75 & 0.84 \\
\hline & SfDL & 625.93 & 31.73 & 91.84 & 1.98 \\
\hline & DfSL & 657.89 & 27.26 & 89.59 & 1.91 \\
\hline & DfDL & 594.31 & 33.8 & 97.08 & 0.97 \\
\hline
\end{tabular}

(Lightwave Technologies). The left index finger was used for a "same" response; and the right index finger, for a "different" response. As soon as the listener responded to each trial (attend-frequency task group mean $=$ $610.40 \mathrm{msec}, S D=268.86 \mathrm{msec}$, range $=208.50-$ 1979.00 msec; attend-location task group mean $=598.80$ msec, $S D=263.31 \mathrm{msec}$, range $=221.40-1985.70 \mathrm{msec}$ ), visual feedback was provided for 500 msec. If they answered correctly, a smiley face was displayed. If they answered incorrectly, the same face was shown with a sad expression. TAiL stimuli were automated and presented using Presentation software (Version 16; Neurobehavioral Systems, Inc.).

From the attend-frequency and attend-location tasks, three measures were calculated: baseline, involuntary orienting (the behavioral cost associated with a change in the task-irrelevant sound feature), and conflict resolution (the behavioral cost associated with processing conflicting sound feature). The baseline measure is calculated for each task type from trials where both sound features, frequency and location, remain constant (i.e., SfSL trials). The involuntary orienting measure is calculated as the difference in RT and accuracy between conditions when the task-irrelevant feature was different between Tone 1 and Tone 2 and when it was constant (see Table 1). The conflict resolution measure is calculated as the difference between trials where both auditory features remain constant or change together (congruent trials: SfSL and DfDL), compared with trials where only one feature changes (incongruent trials: DfSL and SfDL).

\section{Procedure}

Before entering the scanner, the participants completed a practice block for each of the attend-frequency and attendlocation tasks. Each practice block involved five trials, accompanied by on-screen instructions. Participants had to reach $60 \%$ accuracy or more to move onto the full testing

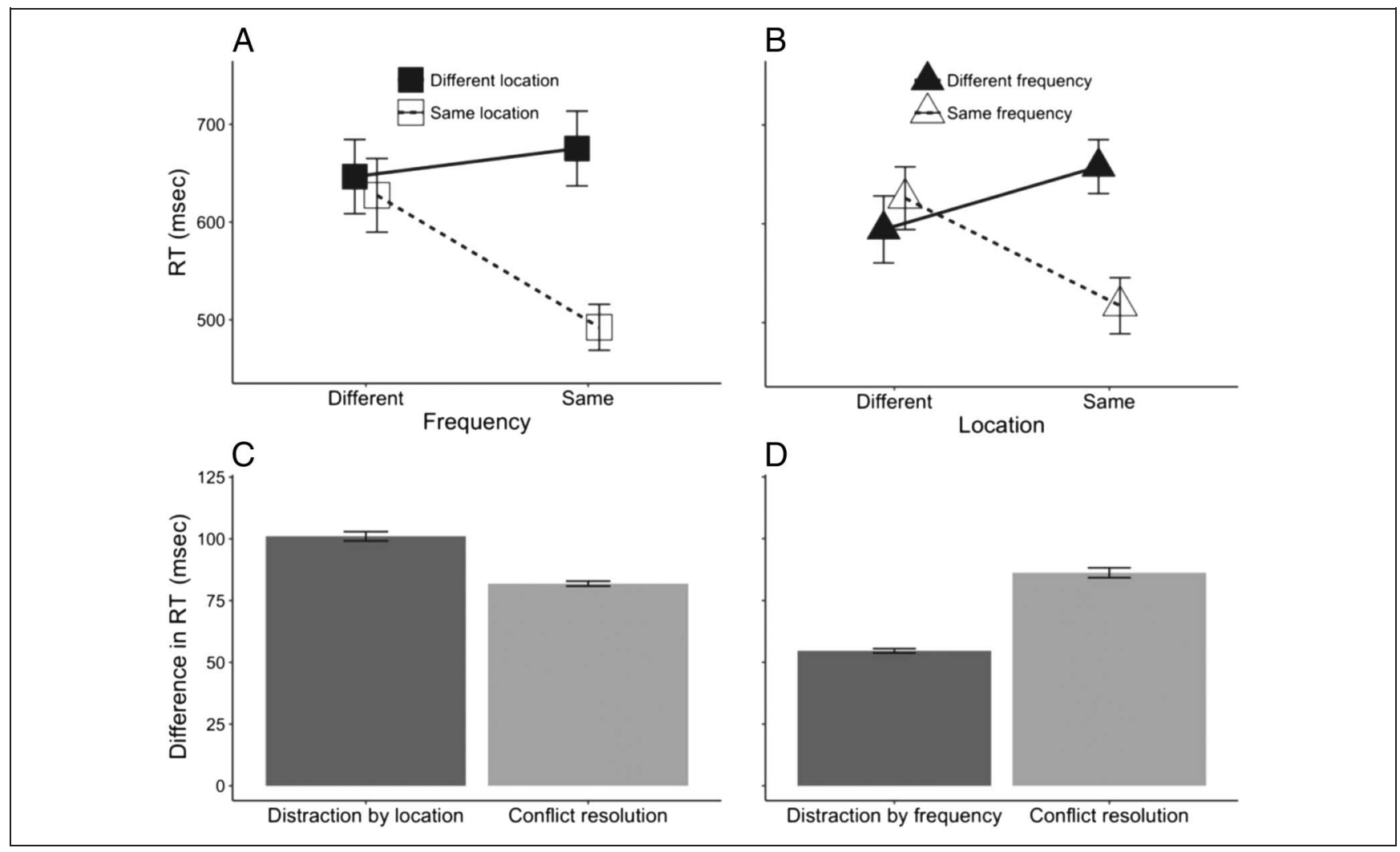

Figure 2. Group mean RTs for the (A) attend-frequency and (B) attend-location tasks. Group mean measures of involuntary orienting and conflict resolution for the (C) attend-frequency and (D) attend-location tasks. Error bars represent SEM. 


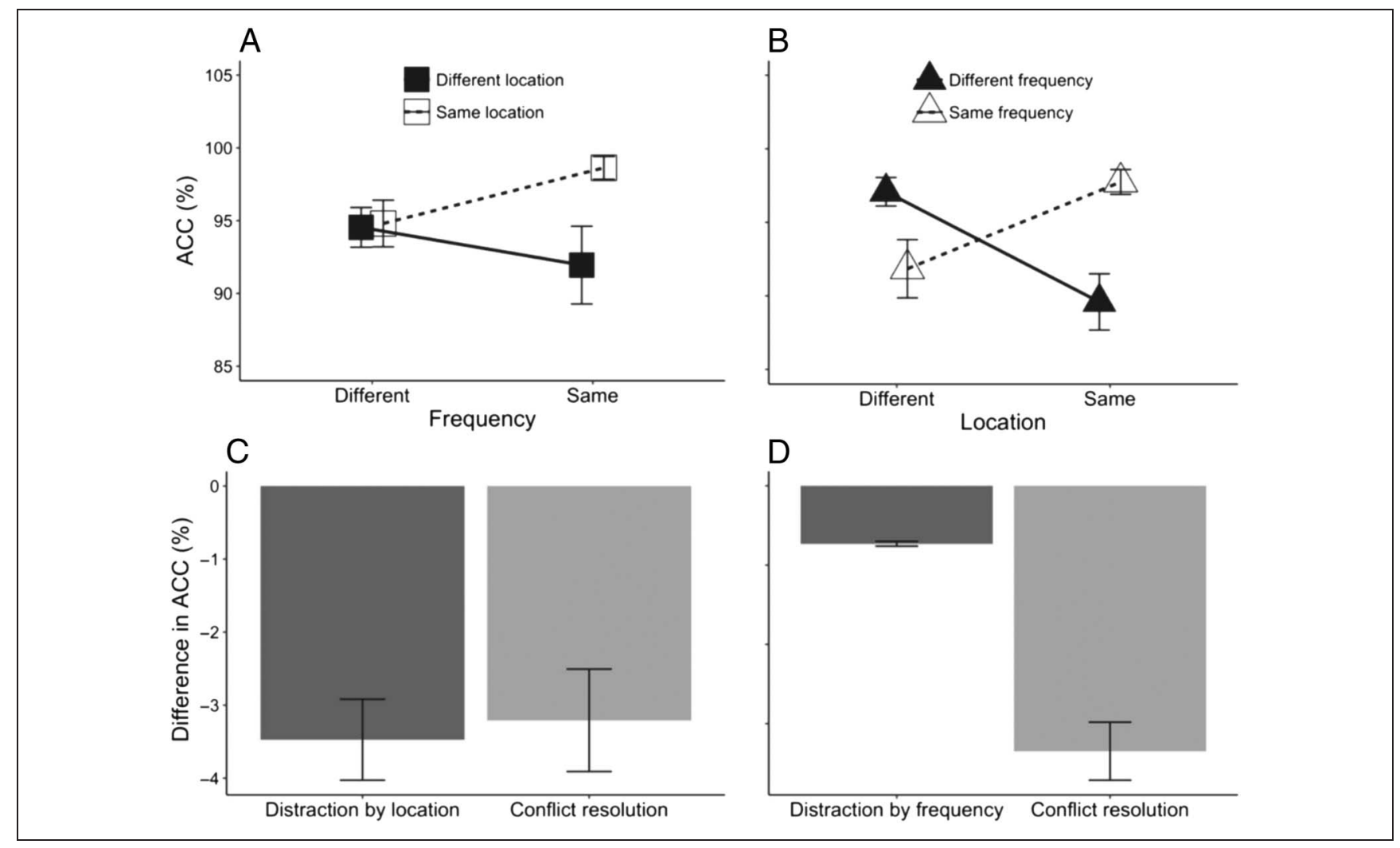

Figure 3. Group mean accuracy for the (A) attend-frequency and (B) attend-location tasks. Group mean measures of involuntary orienting and conflict resolution for the (C) attend-frequency and (D) attend-location tasks. Error bars represent SEM.

blocks in the scanner. Each run consisted of 40 trials10 each of SfSL, DfSL, SfDL, and DfDL-presented in a random order for each participant. Both tasks (attendfrequency and attend-location) had six runs providing 40 trials per task per participant (Figure 1). The order of the task types was counterbalanced across participants and alternated across the runs, allowing regular rests for the participants. At the start of each run, participants were instructed of the task type and to respond as fast and accurately as possible, and visual feedback regarding
Figure 4. Attend-frequency task: Different location versus same location. Surface maps showing the effects of the irrelevant location feature while attending to the relevant frequency feature (i.e., involuntary orienting).

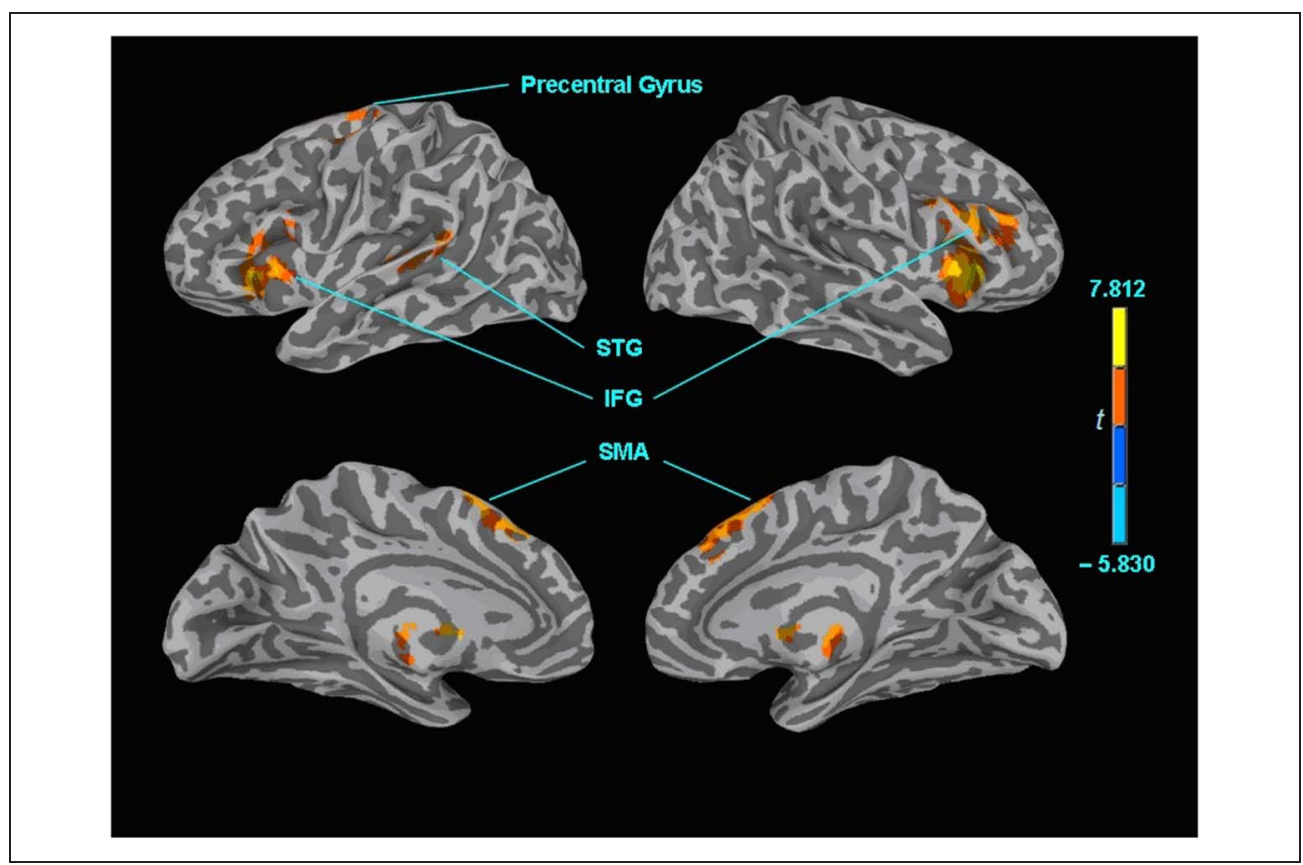


Figure 5. Attend-location task: different frequency versus same frequency. Surface maps showing the effects of the irrelevant frequency feature while attending to the relevant location feature (i.e., involuntary orienting).

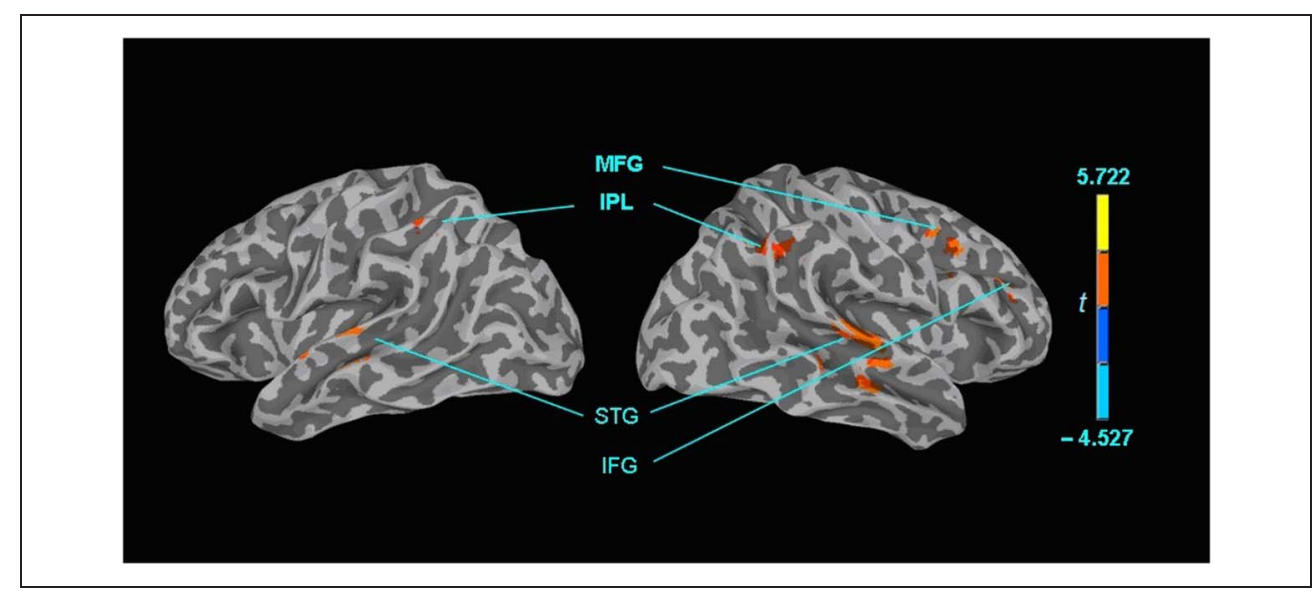

the listener's performance was provided after each trial throughout. The total testing/recording time lasted around $45 \mathrm{~min}$.

\section{Behavioral Analysis}

RTs from correct trials and accuracy (percent correct) were used in the analysis. Repeated-measures ANOVAs with the task-relevant and task-irrelevant features as within-subject factors were run for each TAiL task.

\section{fMRI Scanning and Data Analysis}

Participants were scanned using a research-dedicated whole-body 3.0-T MRI system (Siemens Tim Trio, 3-T software level Syngo MR 2006 VB13T) with a standard 12-channel quadrature bird-cage head coil. Structural T1-weighted anatomical volumes were obtained at the midpoint of the experiment using spoiled gradient recall (axial orientation, repetition time $[\mathrm{TR}]=2000 \mathrm{msec}$, echo time $=2.63 \mathrm{msec}$, field of view $=256 \mathrm{~mm}$, slice thickness $=$ $1 \mathrm{~mm}$ ) for coregistration with the functional images and to ensure that there were no significant brain abnormalities in any of the participants.

Table 3. Attend-Frequency Task

\begin{tabular}{lrrrrr}
\hline & \multicolumn{4}{c}{ Peak MNI Coordinates } & \\
\cline { 2 - 5 } Brain Regions & \multicolumn{1}{c}{ BA } & \multicolumn{1}{c}{$x$} & \multicolumn{1}{c}{$y$} & $z$ & t Values \\
\hline R IFG & 45 & 41 & 25 & -1 & 5.42 \\
L and R SFG & 8 & 3 & 30 & 52 & 5.26 \\
L and R caudate and & & 1 & -26 & -2 & 3.46 \\
$\quad$ commissure & & & & & \\
L IFG & 45 & -33 & 26 & -1 & 5.98 \\
L precentral gyrus & 6 & -28 & -6 & 68 & 3.68 \\
L STG & 22 & -62 & -38 & 22 & 3.34 \\
\hline
\end{tabular}

Irrelevant orienting: Different location versus same location (DfDL + SfDL - DfSL - SfSL; $p t h r=.005, t=3.252$, corrected $p<.05)$.
Each functional scan sequence began with a 20 -sec period where no stimuli were presented. We used an event-related design with a continuous analysis image acquisition. Functional imaging was performed to measure brain activation by means of the BOLD effect (Ogawa, Lee, Kay, \& Tank, 1990). Functional data were acquired using a whole-head T2*-weighted EPI sequence (echo time $=30 \mathrm{msec}$, TR $=$ $2 \mathrm{sec}$, flip angle $=70^{\circ}, 30$ oblique axial slices with interleaved acquisition, $3.125 \mathrm{~mm} \times 3.125 \mathrm{~mm} \times 5 \mathrm{~mm}$ voxel resolution, field of view $=200 \mathrm{~mm}$, acquisition matrix $=$ $64 \times 64)$. Physiological respiratory and cardiac waveforms were recorded from the bellows and photoplethysmograph peripherals on the scanner, respectively, using LabView (National Instruments).

In each run, the first 10 scans were discarded to allow the magnetization to reach steady state. fMRI data were preprocessed and analyzed using Analysis of Functional Neuroimages (AFNI) software (Version AFNI_2011_12_21_1014; Cox, 1996, 2012). In the preprocessing stage, the RETROICOR technique (Glover, Li, \& Ress, 2000) was used to perform physiological noise correction. By performing a slice timing correction, all slices can be aligned to the time of the acquisition for the center slice. (For the Siemens 3-T Trio Scanner at Baycrest, Slice 1 is the center slice for each TR.) To perform

Table 4. Attend-Location Task

\begin{tabular}{lrrrrr}
\hline & \multicolumn{4}{c}{ Peak MNI Coordinates } & \\
\cline { 2 - 5 } Brain Regions & $B A$ & $x$ & $y$ & $z$ & $t$ Values \\
\hline R IFG & 9 & 50 & 12 & 29 & 3.26 \\
R MFG & 9 & 52 & 35 & 25 & 3.75 \\
R STG & 21 & 69 & -25 & -29 & 4.51 \\
R IPL & 40 & 56 & -46 & 44 & 3.50 \\
L STG & 42 & -64 & -25 & -4 & 3.48 \\
L IPL & 40 & -49 & -42 & 57 & 4.06 \\
\hline
\end{tabular}

Irrelevant orienting: Different frequency versus same frequency (DfDL + DfSL - SfDL - SfSL; $p t h r=.005, t=3.252$, corrected $p<.05)$. 
Figure 6. Contrast between the two involuntary orienting effects (attend-frequency > attend-location).

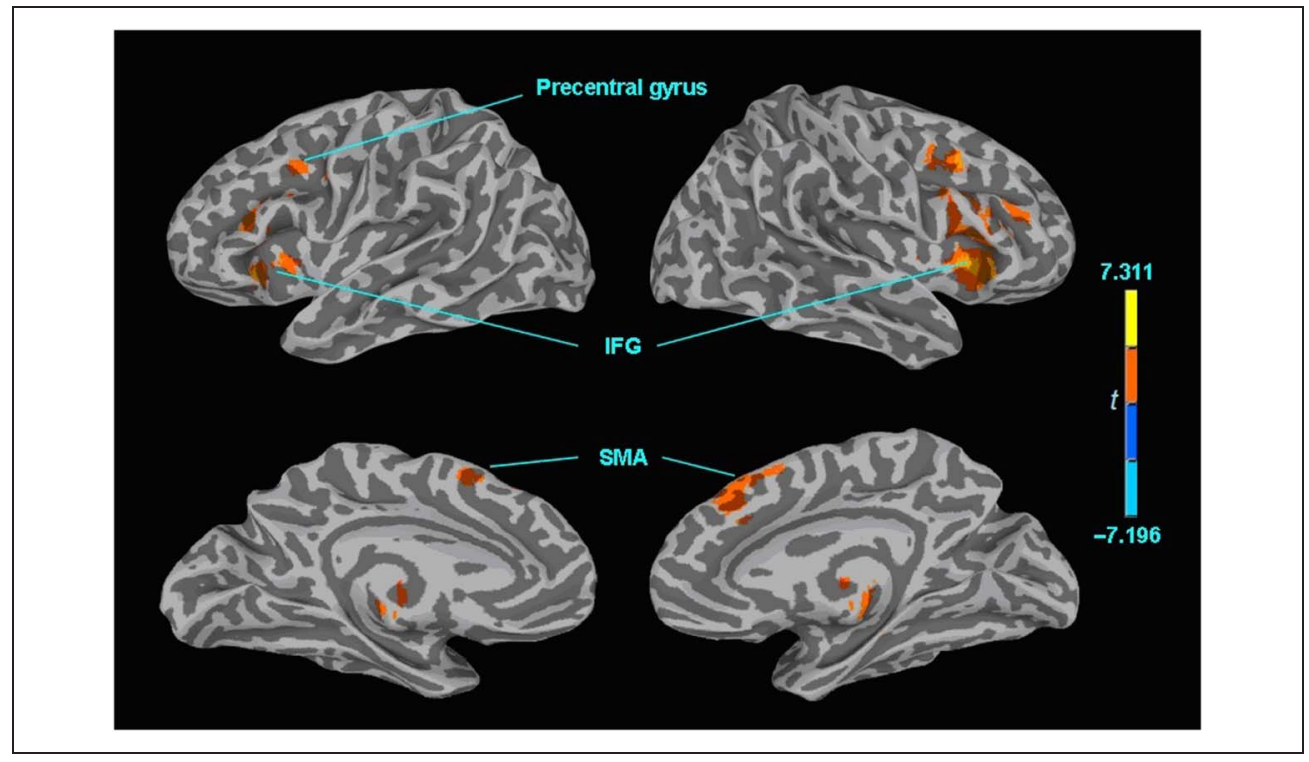

rigid-body motion correction, for each run, images acquired at each point in the series were aligned volumetrically, using the 3 dvolreg plug-in for AFNI, to a reference image acquired during the scanning session. We chose the 51st scan of the middle run (the third run in this study) as a reference scan because the 51st scan is usually very reliable for most data sets. Note that the 51st scan corresponds to Subbrick 50 because the first scan is Subbrick 0. The head motions are captured by six motion parameters: roll = rotation about the I-S axis; pitch = rotation about the R-L axis; yaw = rotation about the A-P axis; $\mathrm{dS}=$ displacement in the Superior direction; $\mathrm{dL}=$ displacement in the left direction; and $\mathrm{dP}=$ displacement in the Posterior direction. The maximum rotation is less than $1.5^{\circ}$, and the peak range of displacement was less than $1.5 \mathrm{~mm}$ for all participants. The coregistration results were also checked visually for additional quality control. To effectively compare fMRI data across participants, baseline normalization was performed by calculating the percent change. The percent signal change is calculated for each participant on a voxel-by-voxel basis. The program 3dAutomask was used to create a mask image to specify what part of the image is of the brain and what part is not. It can eliminate noise outside the brain and reduce the number of voxels.

The program $3 d$ Deconvolve, which provides deconvolution analysis of fMRI time series data on a voxel-by-voxel basis, was performed on the preprocessed data by using

Table 5. Attend-Frequency vs. Attend-Location Task

\begin{tabular}{lccccc}
\hline & \multicolumn{4}{c}{ Peak MNI Coordinates } & \\
\cline { 2 - 5 } Brain Regions & $B A$ & $x$ & $y$ & $z$ & $t$ Values \\
\hline R STG & 22 & 69 & -20 & -7 & -3.97 \\
\hline
\end{tabular}

Involuntary orienting (SfDL $+\mathrm{SfSL}-\mathrm{DfSL}-\mathrm{SfSL} ; p t h r=.005, t=$ 3.252 , corrected $p<.05$ ). a linear fitting. The shape of the response was modeled as a "gamma" function time-locked on trial onset for each stimulus condition. Only trials where participants responded correctly were included in the event-related analysis. For each participant, we used all runs to create activation maps for each of the four conditions: SfSL, DfSL, SfDL, and DfDL. These activation maps were then spatially normalized to a Montreal Neurological Institute (MNI) template. We decreased spatial noise variance by convolving the fMRI brain volume to a 6-mm FWHM smoothness using $3 d F W H M$. The images were also detrended by fitting a thirdorder Legendre polynomial at each voxel and regressing it out of the time series.

AFNI's general linear model (Winkler, Ridgway, Webster, Smith, \& Nichols, 2014) was then used to perform second-level group analysis and create maps to identify statistically significant (threshold $p<.005, F \geq$ 3.252) group effect clusters in the various contrasts. Monte Carlo simulations with AlphaSim in AFNI was run to perform familywise error correction for multiple comparisons (uncorrected $p$ value $=.005$, corrected $p$ value $=.03$, minimum cluster size $=12$, cluster connection radius $=6.67 \mathrm{~mm}$ ); only clusters larger than $586 \mu \mathrm{L}$ were selected. These single-participant univariate maps were then warped to MNI space, using the EPI to MNI transformation that was previously computed for each participant's data. AFNI's Surface Mapping program was used to display the images.

\section{RESULTS}

\section{Baseline}

The group mean accuracy and RTs are presented in Table 2. In trials with no distracting or conflicting auditory information (i.e., SfSL), performance was comparable between the two tasks (attend-frequency and attend-location) for RT, 
Figure 7. Attend-frequency task: incongruent versus congruent. Surface maps showing the effects of incongruency (DfSL + SfDL DfDL - SfSL) while attending to the relevant frequency feature (i.e., conflict resolution).

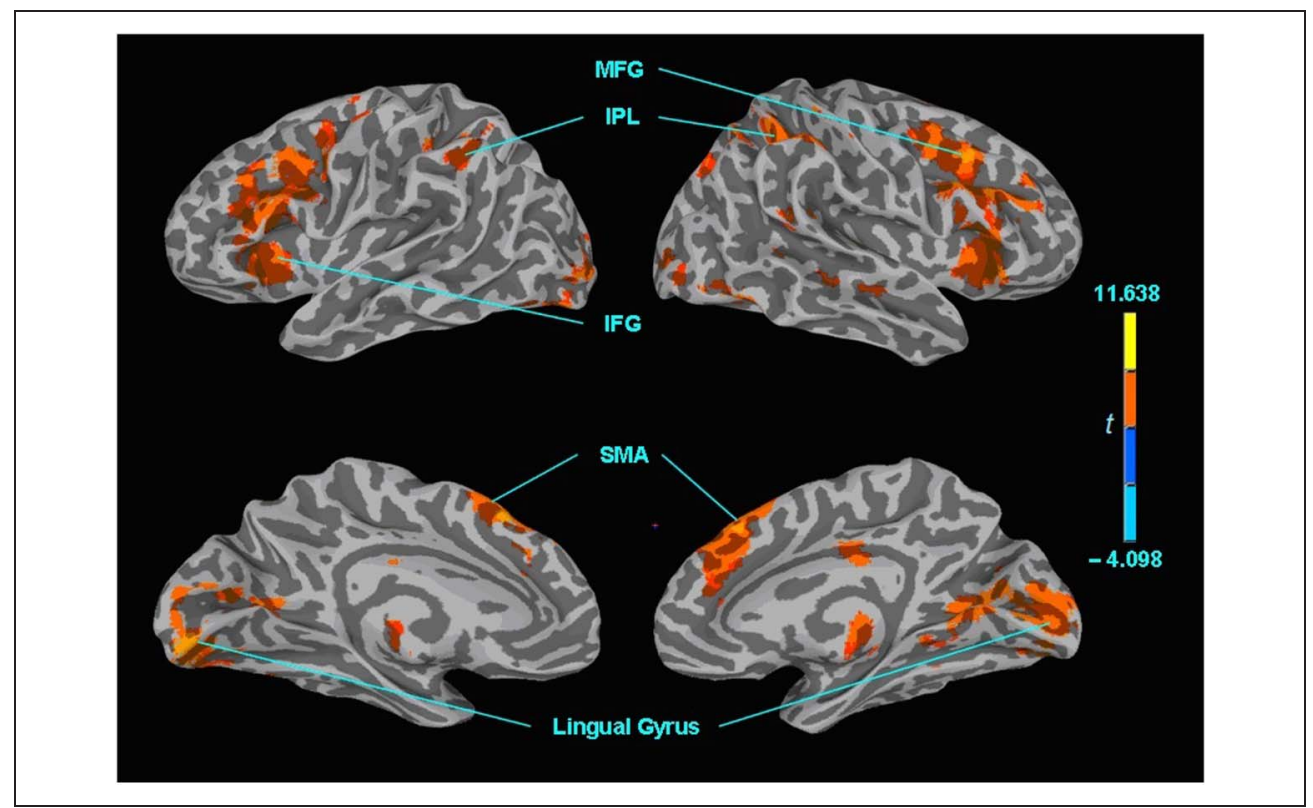

$t(16)=-1.35, p=.20$, and accuracy, $t(16)=1.45, p=.17$. This result suggests that differences, behavioral and cortical, in involuntary orienting and conflict resolution between the two tasks cannot be easily accounted for by differences in task difficulty.

\section{Involuntary Orienting}

\section{Behavioral Results}

A significant effect of distraction (i.e., when the taskirrelevant sound features were different vs. the same) was found in each TAiL task. In the attend-frequency task, participants were significantly slower at responding to trials where the task-irrelevant location of the sounds changed (SfDL and DfDL) compared to when they stayed constant $\left(\right.$ SfSL and DfSL; RT: $F(1,16)=66.64, p<.001, \eta_{\mathrm{p}}^{2}=.81$; Figure 2A). However, they showed no difference in accuracy, $F(1,16)=36.27, p=.097, \eta_{\mathrm{p}}^{2}=.16$ (Figure $\left.3 \mathrm{~A}\right)$. In the attend-location task, listeners were significantly slower and less accurate at responding to trials where the irrelevant frequency of the sounds changed (DFSL and DfDL) compared to when they stayed constant (SfSL and SfDL; RT: $F(1,16)=75.54, p<.001, \eta_{\mathrm{p}}^{2}=.83$; accuracy: $F(1,16)=8.12, p=.012, \eta_{\mathrm{p}}^{2}=.34 ;$ Figures $2 \mathrm{~B}$ and $3 \mathrm{~B})$.

The involuntary orienting difference in RT was significantly lower in the attend-location task compared to the attend-frequency task, $t(16)=3.25, p=.005$ (Figure 2C and D), but not for accuracy, $p=.27$ (Figure 3C and D).

To sum up, in both tasks, RTs were slower when the task-irrelevant feature changed than when it stayed the same, and this effect was greater when attention was focused on nonspatial (frequency) than spatial (location) auditory feature.

\section{fMRI Results}

Figures 4 and 5 show activity associated with involuntary orienting during attend-frequency and attend-location
Figure 8. Attend-location task: incongruent versus congruent. Maps showing the effects of incongruency (DfSL + SfDL DfDL - SfSL) while attending to the relevant location feature (i.e., conflict resolution).

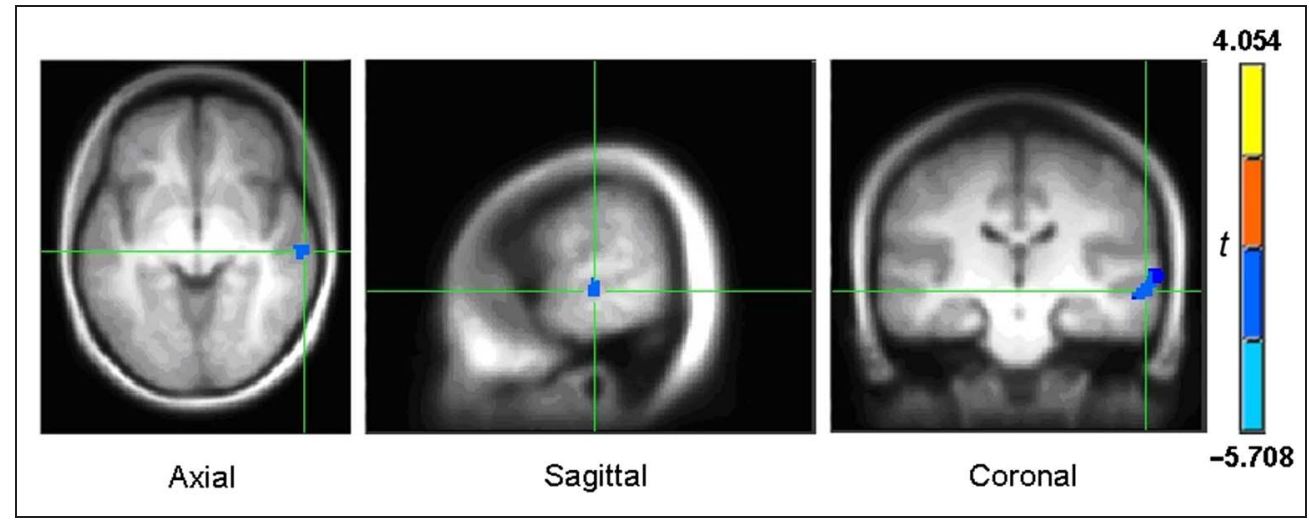


tasks, respectively. As for the behavioral data, the neural correlates were examined by contrasting BOLD responses when the task-irrelevant feature changed versus when the same task-irrelevant feature was repeated within the trial. When participants were instructed to focus attention on frequency, changes in task-irrelevant sound location were associated with increased BOLD signal bilaterally in the superior frontal gyrus (SFG), the right IFG, the left STG, and the left precentral gyrus (Table 3). Conversely, when participants were instructed to focus attention on sound location, changes in task-irrelevant sound frequency were associated with increased BOLD signal in the right MFG, right IFG, bilateral STG, and bilateral inferior parietal lobule (IPL; Table 4).

We also tested whether the changes related to involuntary orienting differed between the attend-frequency and attend-location tasks. The attend-frequency task (SfDLSfSL) minus the attend-location task (DfSL-SfSL) revealed greater activity in the attend-location task in the right STG (BA 22; Figure 6). That is, task-irrelevant changes in sound frequency were associated with enhanced activity in the right STG. Although this difference in activation is relatively small, it suggests that the dorsal and ventral pathways are differentially recruited in situations designed to "trigger" involuntary orienting (Table 5).

\section{Conflict Resolution}

\section{Behavioral Results}

Evidence of conflict resolution was found in each TAiL task. In the attend-frequency task, participants were significantly slower and less accurate at responding to trials with incongruent sound features (SfDL and DfSL) compared to trials with congruent sound features (SfSL and DfDL; RT: $F(1,16)=49.95, p<.001, \eta_{\mathrm{p}}^{2}=.76$; accuracy: $F(1,16)=9.80, p=.006, \eta_{\mathrm{p}}^{2}=.38$; Figures $2 \mathrm{~A}$ and $\left.3 \mathrm{~A}\right)$. Similarly, in the attend-location task, listeners were significantly slower and less accurate at responding to incongruent trials compared to congruent trials (RT: $F(1,16)=$ $113.54, p<.001, \eta_{\mathrm{p}}^{2}=.88$; accuracy: $F(1,16)=21.55, p<$ $.001, \eta_{\mathrm{p}}^{2}=.57$; Figures $2 \mathrm{~B}$ and $3 \mathrm{~B}$ ).

The conflict resolution difference in RT was comparable between the two tasks (attend-frequency and attendlocation), $t(16)=-0.11, p=.91$ (Figure 2C and D), but the difference in accuracy was significantly smaller for the attend-frequency task, $t(16)=2.55, p=.022$ (Figure 3C and D).

\section{fMRI Results}

The neural activity associated with conflict resolution is shown in Figures 7 and 8. As for the behavioral data, the neural correlates were examined by contrasting BOLD responses when one task feature changed (incongruent trials) versus when both of the task features changed or stayed constant within the trial (congruent trials).
Table 6. Attend-Frequency Task

\begin{tabular}{lrrrrr}
\hline & \multicolumn{3}{c}{ Peak MNI Coordinates } & \\
\cline { 2 - 5 } Brain Regions & $B A$ & $x$ & $y$ & $z$ & $t$ Values \\
\hline R IFG & 47 & 52 & 22 & -5 & 3.73 \\
R and L SFG & 6 & 3 & 17 & 53 & 3.73 \\
R cingulate gyrus & 23 & 20 & -5 & 30 & 3.83 \\
R and L culmen & 35 & 18 & -27 & -20 & 4.04 \\
R caudate head & & 16 & 29 & 1 & 3.60 \\
R caudate tail & & 29 & -40 & 21 & 3.45 \\
L precentral gyrus & 9 & -45 & 9 & 34 & 4.15 \\
L IFG & 13 & -37 & 22 & -3 & 5.02 \\
L parahippocampal gyrus & 19 & -37 & -48 & 2 & 3.90 \\
\hline
\end{tabular}

Conflict resolution (DfSL + SfDL - DfDL - SfSL; $p t h r=.005, t=3.252$, corrected $p<.05)$.

When participants were instructed to focus attention on frequency, incongruent trials (DfSL + SfDL) generated a greater BOLD response than congruent trials (DfDL + $\mathrm{SfSL}$ ) in several brain regions. These included bilateral SFG and IFG, MFG, and right ACC as well as right caudate and left parahippocampal gyrus (Table 6).

Conversely, when participants were instructed to focus attention on sound location, the incongruent trials, relative to congruent trials, were associated with increased

Table 7. Attend-Location Task

\begin{tabular}{lrrrrr}
\hline & \multicolumn{3}{c}{ Peak MNI Coordinates } & \\
\cline { 2 - 5 } Brain Regions & $B A$ & $x$ & $y$ & $z$ & $t$ Values \\
\hline R MFG & 9 & 50 & 18 & 33 & 6.09 \\
R STG & 22 & 52 & -22 & -7 & 3.72 \\
R IPL & 40 & 40 & -63 & 56 & 3.72 \\
R inferior semilunar lobule & & 20 & -76 & -50 & 3.60 \\
R cerebellum & & 37 & -46 & -56 & 5.44 \\
R and L SFG & 8 & 1 & 23 & 50 & 4.18 \\
R and L cingulate gyrus & 23 & 1 & -20 & 29 & 3.27 \\
R and L lingual gyrus & 18 & -7 & -89 & -15 & 4.03 \\
R and L posterior commissure & & 1 & -27 & -4 & 3.47 \\
L IFG & 6 & -31 & 26 & -5 & 5.67 \\
L IPL & 40 & -43 & -43 & 46 & 4.72 \\
L inferior semilunar lobule & & -14 & -78 & -47 & 5.85 \\
L cerebellar tonsil & & 1 & -58 & -37 & 4.08 \\
\hline
\end{tabular}

Conflict resolution (DfSL + SfDL - DfDL - SfSL; $p t h r=.005, t=3.252$, corrected $p<.05)$. 
Figure 9. Contrast between the two conflict resolution effects (attend-frequency > attend-location).

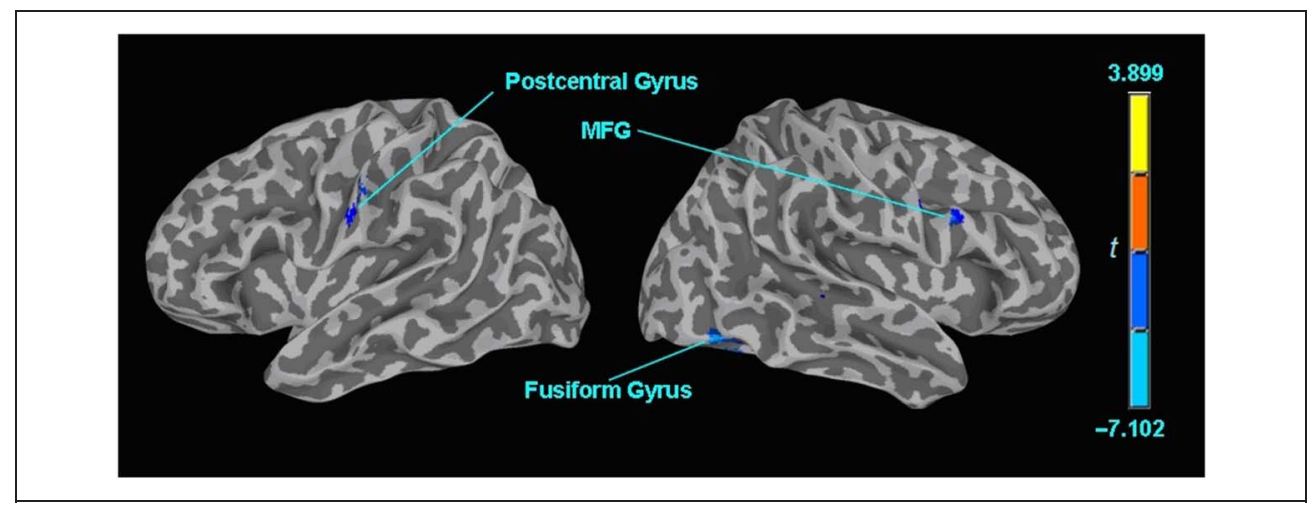

activation in bilateral SFG, right STG, left IFG, left IPL, and cerebellum as well as right MFG, right precuneus, right MTG, and bilateral cingulate (Table 7).

As with involuntary orienting, we tested whether the incongruency effects observed during the attendfrequency and attend-location tasks differed. This contrast revealed greater activation during the location task in the right STG, right MFG, and left postcentral gyrus (Figure 9, Table 8).

\section{DISCUSSION}

As previously found with the TAiL paradigm (Stewart et al., 2017; Zhang et al., 2012), listeners showed a stronger effect of involuntary orienting on RTs in the attend-frequency task than in the attend-location task. Meanwhile, the effect of conflict resolution on RTs was comparable between the two TAiL tasks. These results suggest that, although conflicting auditory information is dealt with similar ease between nonspatial and spatial tasks, participants showed a greater interference when the task-irrelevant sound feature was location than when it was frequency.

Overall, the level of accuracy in both tasks was slightly higher than that in Stewart et al. (2017); however, this may have been because of a shorter paradigm used in this

Table 8. Attend-Frequency vs. Attend-Location Task

\begin{tabular}{lccccc}
\hline & \multicolumn{4}{c}{ Peak MNI Coordinates } & \\
\cline { 2 - 5 } Brain Regions & $B A$ & $x$ & $y$ & $z$ & $t$ Values \\
\hline R fusiform gyrus & 37 & 50 & -69 & -17 & -4.38 \\
R STG & 22 & 52 & -18 & -7 & -3.34 \\
R cerebellar tonsil & & 39 & -48 & -49 & -3.52 \\
R MFG & 9 & 58 & 23 & 35 & -3.27 \\
L postcentral gyrus & 6 & -62 & -16 & 47 & -3.59 \\
L cerebellar tonsil & & -35 & -51 & -51 & -4.61 \\
\hline
\end{tabular}

Conflict resolution $[(\mathrm{DfSL}+\mathrm{SfDL}-\mathrm{DfDL}-\mathrm{SfSL})-(\mathrm{DfSL}+\mathrm{SfDL}-$ DfDL $-\mathrm{SfSL})](p t h r=.005, t=3.252$, corrected $p<.05)$. current study. This replication of behavioral results provides further evidence supporting the use of TAiL as a robust measure of involuntary orienting and conflict resolution in the auditory domain. In this study, both attend-frequency and attend-location tasks showed similar levels of accuracy when no distracting or conflicting sound information was presented. Therefore, difference in brain activity between the two tasks cannot easily be accounted for by differences in task difficulty.

We predicted similar neural networks for involuntary orienting when the auditory features to be attended to were spatial and nonspatial, with additional intraparietal (dorsal stream) areas recruited when attending to spatial stimuli and temporal (ventral stream) areas when attending to nonspatial stimuli. We found that a subset of brain areas was active in the two TAiL tasks covering frontal (SFG, right IFG), parietal (IPL, precentral gyrus), and temporal (STG, MTG) areas.

Activity in the STG was found for involuntary orienting in both TAil tasks. Two meta-analysis studies have shown that this area has been found to be activated for nonspatial and some spatial auditory processing. Arnott et al. (2004) further define nonspatial activations throughout the length of the STG, with spatial activations only occurring in a narrow anterior-posterior range of the cortex. This was later confirmed by Alho et al. (2014) whose meta-analysis found nonspatial pitch processing in the middle STG and spatial auditory processing in the posterior STG. These nonspatial areas are comparable to the temporal regions activated by orienting to a talker after a nonspatial cue in speech-innoise tasks (Lee et al., 2013; Hill \& Miller, 2010).

We found a common frontotemporal network when involuntary orienting in the nonspatial and spatial auditory tasks, similar to results found by Alho et al. (2015) when comparing its nonspatial paradigm to its sister's spatial paradigm (Salmi et al., 2009). We also found additional areas recruited in the nonspatial auditory task. However, instead of activation in the STG, we found additional ventral areas were recruited, including the IFG bilaterally, an area implicated previously during sound object identification tasks including frequency discrimination (Zald \& Pardo, 2002; Kiehl, Laurens, Duty, Forster, \& Liddle, 2001; Müller, Kleinhans, \& Courchesne, 2001), animal noise recognition 
(Tranel et al., 2003), discerning pleasant from unpleasant music (Koelsch, Fritz, von Cramon, Müller, \& Friederici, 2006), and duration discrimination (Pedersen et al., 2000).

Task-specific activations were also found. When comparing between the two TAiL tasks, involuntary orienting was found to show significantly greater activity in the right STG during the attend-location task than the attend-frequency task. This area is generally considered to be part of the ventral network and has been shown through lesion (e.g., Liégeois-Chauvel, Peretz, Babaï, Laguitton, \& Chauvel, 1998; Samson \& Zatorre, 1988; Zatorre, 1985) and fMRI (e.g., Binder et al., 1997; Zatorre, Evans, \& Meyer, 1994) studies to be associated with discerning pitch patterns. The similar involuntary orienting networks with taskspecific areas of activation suggest an interaction between the dorsal and ventral pathways during involuntary orienting. This finding is novel, but the causality of this interaction is unclear. It could be that the interaction between pathways triggers the involuntary orienting, or it may be that the task-irrelevant feature distracts the listener during the task. The right STG activity in the attend-location involuntary orienting contrast suggests the latter.

Braga, Wilson, Sharp, Wise, and Leech (2013) discuss how the FEFs may be part of a superior frontoparietal network used in visual spatial attention, whereas a frontal-temporal network is used for nonspatial auditory attention. This latter network is further described to link the posterior MTG, part of the higher-level auditory cortex, with the "executive" MFG. We did not find this exact network during our auditory task. Instead, we found STG activity related to nonspatial and spatial involuntary orienting and spatial conflict resolution (further details below). Significantly more right STG activity was shown for both attention constructs in our spatial task compared to our nonspatial equivalent. Therefore, our results may extend Braga et al.'s proposal that auditory spatial and nonspatial attention calls upon an MFG/IFG modulator that connects to the MTG/STG and works in parallel to visual attention networks. Unfortunately, Braga et al. (2013) did not have a spatial auditory (or nonspatial visual) task for direct comparisons.

We predicted recruitment of a frontoparietal network for conflict resolution in both TAiL tasks, with activity in ACC, IFG, and parietal areas. Additional frontal activity, as part of the ventral network, was predicted for the nonspatial task. After Posner and Petersen's (1990) assumption that attention networks are amodal, additional activity for the nonspatial task was expected in the occipital lobe, as found in visual studies of conflict resolution (e.g., Siemann et al., 2018). However, as evidence suggests that attention is not amodal (Salo, Salmela, Salmi, Numminen, \& Alho, 2017; Roberts \& Hall, 2008; Salmi, Rinne, Degerman, Salonen, \& Alho, 2007), an alternative prediction was made for additional ventral stream activity in the temporal cortex for the nonspatial task (e.g., Roberts \& Hall, 2008).

As expected, a frontoparietal network was found to be activated for both TAiL tasks' conflict resolution measure. However, ACC, an area typically found in conflict resolution studies, was not included in either network. The spatial task recruited a larger frontoparietal network with additional areas recruited in the fusiform gyrus and dorsal pathway (left postcentral gyrus, right STG, and MFG). IFG areas, typical of the ventral pathway, were recruited for both the spatial and nonspatial tasks. This is consistent with previous conflict resolution studies showing that, when a conflict is detected, a cognitive control system in the dorsolateral pFC is alerted to reduce the conflict by applying favorable weighting to task-relevant information processing to successfully complete the task (Botvinick, Braver, Barch, Carter, \& Cohen, 2001).

Again, this pattern of spatial/nonspatial results differs from those using a common visual paradigm with spatial and nonspatial stimuli. For example, Siemann et al. (2018) found a common visual conflict resolution network for both spatial and nonspatial stimuli, with additional areas recruited for nonspatial stimuli. Meanwhile, our auditory results show that additional dorsal pathway areas were recruited for spatial conflict resolution. Furthermore, our ERP study (Stewart et al., 2017) showed that, although both TAiL tasks had earlier onsets of conflict resolution processing compared to similar visual studies, the auditory spatial task had an additional negative frontocentral component with timings straddling both auditory and visual Stroop tasks. Together with our fMRI findings, this suggests that resolving conflict in auditory spatial tasks is more cortically demanding than in auditory nonspatial tasks.

Our finding is in contrast to the findings of Haupt, Axmacher, Cohen, Elger, and Fell (2009) who found that nonspatial auditory conflict resolution required more activation in the very posterior part of ACC than spatial auditory conflict resolution. However, unlike in TAiL, Haupt et al. (2009) used semantic stimuli to create their auditory Stroop task ("high," "low," and "good") along with the pitch of the stimuli. Whereas semantic stimuli can be processed categorically in such a task, pitch stimuli are not. With the exception of musicians with absolute pitch, most listeners process pure tones on a continuous scale; the typical listener is unable to categorize a frequency of $261.6 \mathrm{~Hz}$ as middle $\mathrm{C}$ and would instead label the tone with an abstract label. This has been shown using another version of an auditory Stroop task where conflict resolution was assessed by congruent and incongruent trials of the stimuli's tone and sung tone name (Schulze, Mueller, \& Koelsch, 2013). Unlike musicians without absolute pitch, those with absolute pitch showed activation in the left STG/STS. This activation of the ventral pathway suggests that only the musicians with absolute pitch were able to categorically perceive/process the tones.

The use of pure tones in this current study can therefore be viewed as favorable as it removes individual differences in their interpretation, unlike semantic stimuli. However, Roebuck, Sindberg, and Weismer (2018) have shown that the interpretation of the executive function inhibition abilities of children with language difficulties changes when nonlinguistic auditory stimuli are familiar (e.g., duck quack 
and dog bark) instead of abstract. Therefore, conclusions about attention constructs from pure tone stimuli should be taken with caution as language strategies may be used to differentiate the stimulus properties. Nevertheless, as the TAiL paradigm uses calculated scores by subtracting one condition from another (e.g., incongruent - congruent), the effects of individual differences in labeling strategies to identify stimuli should be negligible. Furthermore, as the only difference between the two TAiL tasks is the instructions, the results reflect differences in cognitive processing across tasks rather than differences in physical stimuli. Finally, future studies are needed using larger sample sizes to further characterize the connectivity of the neural networks enabling involuntary orienting and conflict resolution when attending to auditory stimuli.

\section{Conclusion}

There is a plethora of evidence showing that both the auditory and visual senses use dorsal and ventral pathways when attending to spatial and nonspatial information, respectively (Alho et al., 2014; Milner \& Goodale, 2008; Arnott et al., 2004). However, the timelines and how these pathways are facilitated with regard to specific attention constructs differ between the domains. Auditory involuntary orienting and conflict resolution have been found to occur faster than visual involuntary orienting and conflict resolution (Stewart et al., 2017). The results from this current study suggest that additional dorsal pathway cortical areas are recruited for auditory spatial attention constructs. These results are in contrast to the visual modality, where additional cortical areas are recruited for visual nonspatial attention constructs. These differences reemphasize that not all findings in the visual domain can be generalized to audition. Adding to existing evidence from an EEG study, our results suggest that the cognitive processes that occur when selectively attending to auditory stimuli are distinct from visual stimuli and that involuntary orienting occurs before conflict resolution.

\section{Acknowledgments}

We thank Jeff Wong for technical support. This research was supported by grants from the Canadian Institutes of Health Research (MOP 106619), the Natural Sciences and Engineering Research Council of Canada to C. A., and an Erasmus Mundus Exchange Network in Auditory Cognitive Neuroscience award to H. J. S

Reprint requests should be sent to Claude Alain, Rotman Research Institute, Baycrest Centre, 3560 Bathurst Street, Toronto, ON M6A 2E1, Canada, or via e-mail: calain@research.baycrest.org.

\section{REFERENCES}

Alain, C., \& Arnott, S. R. (2000). Selectively attending to auditory objects. Frontiers in Bioscience, 5, D202-D212.

Alain, C., Arnott, S. R., Hevenor, S., Graham, S., \& Grady, C. L. (2001). "What" and "where" in the human auditory system.
Proceedings of the National Academy of Sciences, U.S.A., 98, 12301-12306.

Alain, C., He, Y., \& Grady, C. (2008). The contribution of the inferior parietal lobe to auditory spatial working memory. Journal of Cognitive Neuroscience, 20, 285-295.

Alho, K., Rinne, T., Herron, T. J., \& Woods, D. L. (2014). Stimulus-dependent activations and attention-related modulations in the auditory cortex: A meta-analysis of fMRI studies. Hearing Research, 307, 29-41.

Alho, K., Salmi, J., Koistinen, S., Salonen, O., \& Rinne, T. (2015). Top-down controlled and bottom-up triggered orienting of auditory attention to pitch activate overlapping brain networks. Brain Research, 1626, 136-145.

Arnott, S. R., Binns, M. A., Grady, C. L., \& Alain, C. (2004). Assessing the auditory dual-pathway model in humans. Neuroimage, 22, 401-408.

Binder, J. R., Frost, J. A., Hammeke, T. A., Cox, R. W., Rao, S. M., \& Prieto, T. (1997). Human brain language areas identified by functional magnetic resonance imaging. Journal of Neuroscience, 17, 353-362.

Botvinick, M. M., Braver, T. S., Barch, D. M., Carter, C. S., \& Cohen, J. D. (2001). Conflict monitoring and cognitive control. Psychological Review, 108, 624-652.

Braga, R. M., Wilson, L. R., Sharp, D. J., Wise, R. J. S., \& Leech, R. (2013). Separable networks for top-down attention to auditory non-spatial and visuospatial modalities. Neuroimage, 74, 77-86.

Clarke, S., Bellmann, A., Meuli, R. A., Assal, G., \& Steck, A. (2000). Auditory agnosia and auditory spatial deficits following left hemispheric lesions: Evidence for distinct processing pathways. Neuropsychologia, 38, 797-807.

Clarke, S., \& Thiran, A. B. (2004). Auditory neglect: What and where in auditory space. Cortex, 40, 291-300.

Clarke, S., Thiran, A. B., Maeder, P., Adriani, M., Vernet, O., Regli, L., et al. (2002). What and where in human audition: Selective deficits following focal hemispheric lesions. Experimental Brain Research, 147, 8-15.

Cox, R. W. (1996). AFNI: Software for analysis and visualization of functional magnetic resonance neuroimages. Computers and Biomedical Research, 29, 162-173.

Cox, R. W. (2012). AFNI: What a long strange trip it's been. Neuroimage, 62, 743-747.

Degerman, A., Rinne, T., Salmi, J., Salonen, O., \& Alho, K. (2006). Selective attention to sound location or pitch studied with fMRI. Brain Research, 1077, 123-134

Du, Y., He, Y., Arnott, S. R., Ross, B., Wu, X., Li, L., et al. (2015). Rapid tuning of auditory "what" and "where" pathways by training. Cerebral Cortex, 25, 496-506.

Glover, G. H., Li, T.-Q., \& Ress, D. (2000). Image-based method for retrospective correction of physiological motion effects in fMRI: RETROICOR. Magnetic Resonance in Medicine, 44, 162-167.

Haupt, S., Axmacher, N., Cohen, M. X., Elger, C. E., \& Fell, J. (2009). Activation of the caudal anterior cingulate cortex due to task-related interference in an auditory Stroop paradigm. Human Brain Mapping, 30, 3043-3056.

Hill, K. T., \& Miller, L. M. (2010). Auditory attentional control and selection during cocktail party listening. Cerebral Cortex, 20, 583-590.

Jensen, J. K., \& Neff, D. L. (1993). Development of basic auditory discrimination in preschool children. Psychological Science, 4, 104-107.

Kiehl, K. A., Laurens, K. R., Duty, T. L., Forster, B. B., \& Liddle, P. F. (2001). Neural sources involved in auditory target detection and novelty processing: An event-related fMRI study. Psychophysiology, 38, 133-142.

Killion, M. C., Niquette, P. A., Gudmundsen, G. I., Revit, L. J., \& Banerjee, S. (2004). Development of a quick speech-in-noise test for measuring signal-to-noise ratio loss in normal-hearing 
and hearing-impaired listeners. Journal of the Acoustical Society of America, 116, 2395-2405.

Koelsch, S., Fritz, T., von Cramon, D. Y., Müller, K., \& Friederici, A. D. (2006). Investigating emotion with music: An fMRI study. Human Brain Mapping, 27, 239-250.

Lee, A. K. C., Rajaram, S., Xia, J., Bharadwaj, H., Larson, E., Hämäläinen, M. S., et al. (2013). Auditory selective attention reveals preparatory activity in different cortical regions for selection based on source location and source pitch. Frontiers in Neuroscience, 6, 190.

Leung, A. W. S., \& Alain, C. (2011). Working memory load modulates the auditory "what" and "where" neural networks. Neuroimage, 55, 1260-1269.

Liégeois-Chauvel, C., Peretz, I., Babaï, M., Laguitton, V., \& Chauvel, P. (1998). Contribution of different cortical areas in the temporal lobes to music processing. Brain, 121, 1853-1867.

Lomber, S. G., \& Malhotra, S. (2008). Double dissociation of 'what' and 'where' processing in auditory cortex. Nature Neuroscience, 11, 609-616.

Maeder, P. P., Meuli, R. A., Adriani, M., Bellmann, A., Fornari, E., Thiran, J.-P., et al. (2001). Distinct pathways involved in sound recognition and localization: A human fMRI study. Neuroimage, 14, 802-816.

Mayer, A. R., Franco, A. R., \& Harrington, D. L. (2009). Neuronal modulation of auditory attention by informative and uninformative spatial cues. Human Brain Mapping, 30, $1652-1666$

Mayer, A. R., Harrington, D., Adair, J. C., \& Lee, R. (2006). The neural networks underlying endogenous auditory covert orienting and reorienting. Neuroimage, 30, 938-949.

Milner, A. D., \& Goodale, M. A. (2008). Two visual systems re-viewed. Neuropsychologia, 46, 774-785.

Müller, R.-A., Kleinhans, N., \& Courchesne, E. (2001). Broca's area and the discrimination of frequency transitions: A functional MRI study. Brain and Language, 76, 70-76.

Ogawa, S., Lee, T. M., Kay, A. R., \& Tank, D. W. (1990). Brain magnetic resonance imaging with contrast dependent on blood oxygenation. Proceedings of the National Academy of Sciences, U.S.A., 87, 9868-9872.

Orr, J. M., \& Weissman, D. H. (2009). Anterior cingulate cortex makes 2 contributions to minimizing distraction. Cerebral Cortex, 19, 703-711.

Pedersen, C. B., Mirz, F., Ovesen, T., Ishizu, K., Johannsen, P., Madsen, S., et al. (2000). Cortical centres underlying auditory temporal processing in humans: A PET study. Audiology, 39, 30-37.

Petersen, S. E., \& Posner, M. I. (2012). The attention system of the human brain: 20 years after. Annual Review of Neuroscience, 35, 73-89.

Posner, M. I., \& Petersen, S. E. (1990). The attention system of the human brain. Annual Review of Neuroscience, 13, 25-42.

Rauschecker, J. P. (1998). Parallel processing in the auditory cortex of primates. Audiology and Neurotology, 3, 86-103.

Rauschecker, J. P., \& Tian, B. (2000). Mechanisms and streams for processing of "what" and "where" in auditory cortex. Proceedings of the National Academy of Sciences, U.S.A., 97, 11800-11806.

Roberts, K. L., \& Hall, D. A. (2008). Examining a supramodal network for conflict processing: A systematic review and novel functional magnetic resonance imaging data for related visual and auditory Stroop tasks. Journal of Cognitive Neuroscience, 20, 1063-1078.

Roebuck, H., Sindberg, H., \& Weismer, S. E. (2018). The role of language in nonlinguistic stimuli: Comparing inhibition in children with language impairment. Journal of Speech, Language, and Hearing Research, 61, 1216-1225.

Romanski, L. M., Tian, B., Fritz, J., Mishkin, M., Goldman-Rakic, P. S., \& Rauschecker, J. P. (1999). Dual streams of auditory afferents target multiple domains in the primate prefrontal cortex. Nature Neuroscience, 2, 1131-1136.

Salmi, J., Rinne, T., Degerman, A., Salonen, O., \& Alho, K. (2007). Orienting and maintenance of spatial attention in audition and vision: Multimodal and modality-specific brain activations. Brain Structure and Function, 212, 181-194.

Salmi, J., Rinne, T., Koistinen, S., Salonen, O., \& Alho, K. (2009). Brain networks of bottom-up triggered and top-down controlled shifting of auditory attention. Brain Research, 1286, 155-164.

Salo, E., Salmela, V., Salmi, J., Numminen, J., \& Alho, K. (2017). Brain activity associated with selective attention, divided attention and distraction. Brain Research, 1664, 25-36.

Samson, S., \& Zatorre, R. J. (1988). Melodic and harmonic discrimination following unilateral cerebral excision. Brain and Cognition, 7, 348-360.

Schulze, K., Mueller, K., \& Koelsch, S. (2013). Auditory Stroop and absolute pitch: An fMRI study. Human Brain Mapping, 34, 1579-1590.

Siemann, J., Herrmann, M., \& Galashan, D. (2018). The effect of feature-based attention on flanker interference processing: An fMRI-constrained source analysis. Scientific Reports, 8, 1580.

Stewart, H. J., \& Amitay, S. (2015). Modality-specificity of selective attention networks. Frontiers in Psychology, 6, 1826.

Stewart, H. J., Amitay, S., \& Alain, C. (2017). Neural correlates of distraction and conflict resolution for nonverbal auditory events. Scientific Reports, 7, 1595.

Stroop, J. R. (1935). Studies of interference in serial verbal reactions. Journal of Experimental Psychology, 18, 643-662.

Tranel, D., Damasio, H., Eichhorn, G. R., Grabowski, T., Ponto, L. L. B., \& Hichwa, R. D. (2003). Neural correlates of naming animals from their characteristic sounds. Neuropsychologia, 41, 847-854.

Winkler, A. M., Ridgway, G. R., Webster, M. A., Smith, S. M., \& Nichols, T. E. (2014). Permutation inference for the general linear model. Neuroimage, 92, 381-397.

Zald, D. H., \& Pardo, J. V. (2002). The neural correlates of aversive auditory stimulation. Neuroimage, 16, 746-753.

Zatorre, R. J. (1985). Discrimination and recognition of tonal melodies after unilateral cerebral excisions. Neuropsychologia, 23, 31-41.

Zatorre, R. J., Evans, A. C., \& Meyer, E. (1994). Neural mechanisms underlying melodic perception and memory for pitch. Journal of Neuroscience, 14, 1908-1919.

Zhang, Y.-X., Barry, J. G., Moore, D. R., \& Amitay, S. (2012). A new test of attention in listening (TAIL) predicts auditory performance. PLoS One, 7, e53502.

Zündorf, I. C., Lewald, J., \& Karnath, H.-O. (2016). Testing the dual-pathway model for auditory processing in human cortex. Neuroimage, 124, 672-681. 